\title{
Unique requirements for retinoid-dependent transcriptional activation by the orphan receptor LXR
}

\author{
Patricia J. Willy and David J. Mangelsdorf ${ }^{1}$ \\ Howard Hughes Medical Institute and the Department of Pharmacology, University of Texas Southwestern Medical Center, \\ Dallas, Texas 75235-9050 USA
}

LXR is an orphan nuclear receptor that confers retinoid responsiveness to the retinoid $X$ receptor (RXR) by its interaction on a specific response element called an LXRE. To understand the mechanism of this response, three characteristics were identified that are crucial to activation of the RXR-LXR complex. First, the orientation of the RXR-LXR heterodimer on DNA indicates that as the ligand-binding partner, RXR occupies the $5^{\prime}$ half-site of the response element. Next, the sequence specificity of the LXRE was determined in order to identify residues required for retinoid activation of the heterodimer. Remarkably, subtle changes in the nucleotide sequence of the LXRE half-sites that do not substantially alter DNA binding of the RXR-LXR heterodimer have a significant effect on the ability of the complex to be activated by ligand. Finally, we characterized the contributions of the activation domains of each receptor to the trans-activation potential of the RXR-LXR heterodimer. Surprisingly, our results show that only the activation domain of LXR is required for retinoid activation. Taken together, these results demonstrate the existence of a unique form of communication between heterodimer partners in which the activation potential of one receptor (LXR) is enabled by ligand binding to its partner (RXR). Furthermore, we conclude that RXR ligand activation potential is not dictated solely by its position on DNA, but is influenced by other factors such as the receptor partner and sequence of the response element.

[Key Words: RXR; LXR; retinoid receptors; heterodimers; trans-activation domain; nuclear receptors]

Received July 26, 1996; revised version accepted December 9, 1996.

Members of the nuclear hormone receptor superfamily, including the receptors for retinoic acid, vitamin D, thyroid hormone, and steroid hormones, are transcription factors that influence a variety of cellular processes in response to ligand binding (for review, see Mangelsdorf et al. 1995). These receptors act by binding to specific cis-acting elements in the promoters of target genes, thereby repressing or activating gene expression. A subgroup of receptors within the superfamily, including the retinoic acid receptor $(\mathrm{RAR})$, thyroid hormone receptor (TR), vitamin $\mathrm{D}$ receptor (VDR), peroxisome proliferator activated receptor (PPAR), and many orphan receptors, bind to specific DNA sequences known as hormone response elements as heterodimers with the retinoid $\mathrm{X}$ receptor (RXR) (for review, see Mangelsdorf and Evans 1995). The hormone response elements generally consist of two direct repeat half-sites of a consensus hexameric sequence (AGGTCA). Response element specificity resides in subtle differences in the sequence of the halfsites as well as the number of nucleotides in the spacer region between the two half-sites. Receptors that bind

${ }^{1}$ Corresponding author.

E-MAIL MANGELSD@UTSW.SWMED.EDU; FAX (214) 648-5068.
DNA as heterodimers with RXR exhibit a specific polarity on direct-repeat response elements such that the silent, non-ligand-binding partner (RXR) occupies the $5^{\prime}$ half-site whereas the ligand-binding receptor (i.e. RAR, $\mathrm{TR}$, or VDR) occupies the $3^{\prime}$ half-site of the response element (Kurokawa et al. 1993; Perlmann et al. 1993; Schräder et al. 1995). These data have led to the suggestion that the position of the receptor on DNA (i.e., binding to the $3^{\prime}$ half-sitel enables the receptor to be activated by ligand.

Nuclear receptors share a common structure consisting of several modular domains. Along with the highly conserved DNA-binding domain and a carboxy-terminal domain that specifies both ligand binding and receptor dimerization, these receptors also contain regions required for transcriptional activation. The amino-terminal region of most of these receptors contains a transcriptional activation domain referred to as AF- 1 that contributes to constitutive activation by the receptor (Hollenberg and Evans 1988; Nagpal et al. 1992). A second transcriptional activation domain termed the AF-2 is located in the carboxyl terminus, but unlike the AF-1 domain, the AF-2 is ligand-dependent and conserved among members of the nuclear hormone receptor super- 
family (Danielian et al. 1992). Mutations within this AF-2 region have been shown to abolish ligand-activated transcription in several receptors including the glucocorticoid receptor, estrogen receptor, RAR, TR, and RXR (Hollenberg and Evans 1988; Webster et al. 1988; Nagpal et al. 1993; Saatcioglu et al. 1993; Barettino et al. 1994; Durand et al. 1994; Tone et al. 1994; Zhang et al. 1994; Baniahmad et al. 1995; Leng et al. 1995). Transcriptional activation by these receptors is thought to involve association with a class of proteins referred to as coactivators that enhance the transcriptional response by interacting with the basal transcriptional machinery (McKnight 1996). Recently, several putative coactivators have been identified that associate with nuclear receptors in a ligand-dependent manner (Halachmi et al. 1994; Cavaillès et al. 1995; Lee et al. 1995; Le Douarin et al. 1995; Oñate et al. 1995; vom Baur et al. 1996). Along with transcriptional activation, TR and RAR recently have been shown to interact with corepressors that mediate repression of target genes in the absence of ligand binding (Chen and Evans 1995; Hörlein et al. 1995).

LXR is an orphan receptor that heterodimerizes with RXR and binds to a specific response element called the LXRE (Willy et al. 1995). The unique association between RXR and LXR on this element allows the complex to be activated by the RXR ligand 9-cis retinoic acid (9cRA). To understand the mechanism by which LXR enables RXR ligand activation, we examined several parameters that are crucial to nuclear receptor heterodimer action. Taken together, our results define the requisite contributions made by RXR, LXR, and the LXRE to the transcriptionally active heterodimer. We find that in contrast to other RXR heterodimers, ligand activation occurs while the ligand-binding receptor (RXR) occupies the $5^{\prime}$ half-site of the response element. In addition, we find that ligand binding by one receptor (RXR) confers activation through the AF-2 domain of the other receptor (LXR). These data suggest the existence of a novel signaling mechanism between heterodimeric partners.

\section{Results}

\section{$R X R-L X R$ heterodimer polarity}

Previous studies have shown that RXR is the ligandbinding partner in the retinoid-activated RXR-LXR heterodimer (Willy et al. 1995). Therefore, it was of interest to determine whether the polarity of the RXR-LXR heterodimer was different from that of other heterodimers in which it is known that the ligand-binding partner occupies the $3^{\prime}$ half-site of the response element and RXR, which does not bind ligand, occupies the $5^{\prime}$ half-site (Kurokawa et al. 1993; Perlmann et al. 1993). To deduce the polarity of the RXR-LXR heterodimer on the LXRE sequence, we used site-directed mutagenesis to alter the amino acids in the DNA-binding domain P box of LXR to those of the glucocorticoid receptor (GR) (Perlmann et al. 19931, thereby changing LXR's half-site binding specificity to AGAACA. The mutant receptor, referred to as LXRpg, was used in electrophoretic mobility shift assays
(EMSA) and transient transfections to ascertain the polarity of binding of the RXR-LXR heterodimer using two mutant LXRE sequences (Fig. 1A). The two mutant sequences, designated G4L and $\mathrm{L} 4 \mathrm{G}$, have their $5^{\prime}$ or $3^{\prime}$ half-site nucleotides changed to those that specify GR binding (Strähle et al. 1987). As shown in Figure 1B, none of the receptors alone could bind to either element; however, in the presence of RXR, LXRpg bound the L4G element in which the GR-specific half-site is in the $3^{\prime}$ position (lane 5). In contrast, no binding was observed with the G4L element using any of the receptor combinations.

To confirm that the in vitro binding of RXR-LXRpg to the L4G response element was functional, LXRpg was tested for ligand-dependent transcriptional activation in

A

$$
\begin{aligned}
& \text { LXRE tgcggttcccag } \overrightarrow{\text { GGTTTA }} \text { aata } \overrightarrow{\text { AGTTCA }} \text { tctagat } \\
& \text { L4G tgcggttcccag GGTTTA aata AGAACA]tctagat } \\
& \text { G4L tgcggttcccag AGAACA a a a AGTTCA tctagat }
\end{aligned}
$$

B

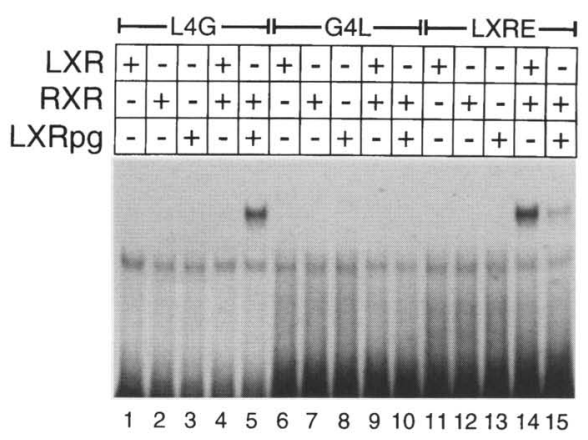

C

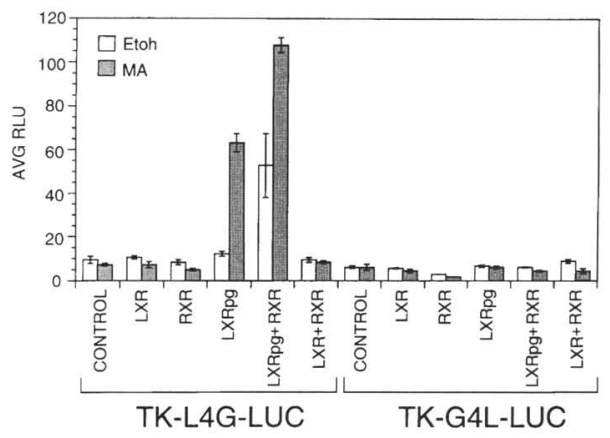

Figure 1. Polarity of the RXR-LXR heterodimer on DNA. $(A)$ Sense-strand sequences of double-stranded oligonucleotides used to demonstrate RXR-LXR polarity. The glucocorticoid receptor-specific half-site is outlined. $(B)$ EMSA analysis using in vitro synthesized receptor proteins in the combinations shown above the gel with one of the three ${ }^{32} \mathrm{P}$-labeled oligonucleotide probes shown in $A$. (C) Transient transfections in CV-1 cells using LXR, RXR, or LXRpg and luciferase reporters containing three copies of either $\mathrm{L} 4 \mathrm{G}$ or G4L response elements shown in $A$. In transfections containing two receptors, equivalent amounts of both receptor plasmids were added to the transfections. (Open bars) Etoh; (shaded bars) methoprene acid (MA); $(\mathrm{RLU})$ relative light units. 
transient transfections of $\mathrm{CV}-1$ cells using a luciferase reporter containing three copies of either the L4G or G4L response element upstream of a minimal promoter (TKL4G-LUC and TK-G4L-LUC, respectively). The RXRselective ligand, methoprene acid (Harmon et al. 1995), which has been shown previously to activate the RXRLXR heterodimer (Willy et al. 1995), was used to activate transcription of the reporter in this and subsequent experiments. Consistent with the in vitro binding results, LXRpg-dependent transcription of the reporter gene occurred with the L4G, but not the G4L reporter (Fig. 1C). Furthermore, when exogenous RXR was added to the transient transfections along with LXRpg, an increase in both basal and ligand-activated transcription was observed (Fig. 1C), which is characteristic of the LXR response on the LXRE in the presence of exogenous RXR. Taken together these results demonstrate that the RXRLXR heterodimer binds to the LXRE with RXR occupying the $5^{\prime}$ half-site and LXR the $3^{\prime}$ half-site. This is the same polarity observed with other RXR heterodimers including RAR, TR, and VDR (Kurokawa et al. 1993; Perlmann et al. 1993; Schräder et al. 1995). However, in contrast to our results with LXR, in other RXR heterodimers it has been shown that ligand must first bind to the 3' partner to initiate trans-activation (Forman et al. 1995; Roy et al. 1995). The fact that RXR directs ligand-dependent transactivation while maintaining its $5^{\prime}$ position within the LXR heterodimer is clear evidence that the orientation of the receptor on DNA alone does not dictate a receptor's ability to be activated by ligand.

\section{Identification of critical nucleotides in the LXRE sequence that specify DNA binding and retinoid-induced transcriptional activation}

Previous studies have shown that the sequence of the LXRE is essential for retinoid activation of the RXR-LXR heterodimer (Willy et al. 1995). The LXRE sequence has been defined as a degenerate DR4-type element containing nucleotide substitutions in both half-sites that deviate from the canonical AGGTCA motif (Willy et al. 1995). To determine which nucleotides are essential and/or optimal for retinoid induced transcriptional activation, numerous substitutions were made in the LXRE sequence. Because we have shown previously that the RXR-LXR heterodimer can not trans-activate all DNA sequences that it binds to with high affinity, our strategy for analyzing LXRE sequences was based on activation rather than DNA binding. Thus, a single copy of each mutated response element was cloned into a luciferase reporter and the ability to confer retinoid activation by LXR was tested in transient transfections of CV-1 cells. Sequences and activation data from these experiments are summarized in Figure 2.

Initially, the $5^{\prime}$ half-site was mutated to make it more closely resemble the consensus AGGTCA sequence. The most notable deviation of the $5^{\prime}$ half-site from the consensus sequence is that the highly conserved $C$ residue normally found in position 5 of the consensus half-site is a $T$ in the LXRE sequence. When this $\mathrm{T}$ was changed to

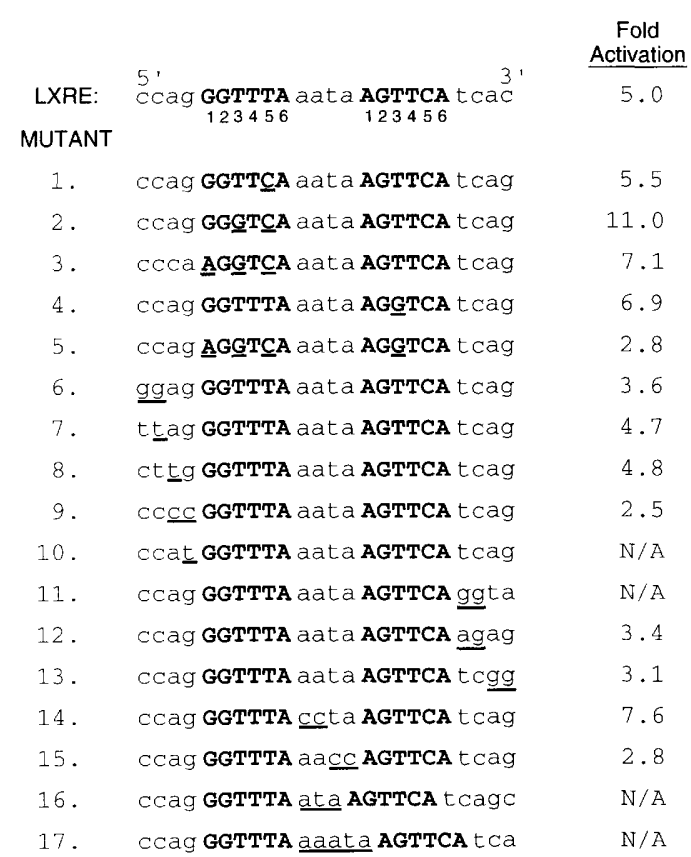

Figure 2. Multiple sequences can function as LXREs. Shown are the sense-strand sequences of the wild-type and mutant LXRE double-stranded oligonucleotides that were cloned as single copies into the TK-LUC reporter. Nucleotides altered from the original LXRE sequence are underlined. Each response element cloned into TK-LUC was tested for LXR activation in transient transfections in $\mathrm{CV}-1$ cells. Fold activation by methoprene acid is given next to each response element sequence. All data were generated from the same experiment performed in triplicate. Nucleotides in the half-sites are numbered and shown in bold. (N/A) Not active.

C, activation by the element was similar to that of the original LXRE (Fig. 2, sequence 1). However, when this mutation was coupled with a $T$ to $G$ conversion at position 3 in the $5^{\prime}$ half-site, generating a GGGTCA sequence, there was a twofold increase in activation on the element (Fig. 2, sequence 2). Interestingly, this is the best retinoid-inducible LXRE we have tested to date. Altering either the $5^{\prime}$ (sequence 3) or $3^{\prime}$ (sequence 4) half-site alone to give the consensus AGGTCA sequence also resulted in activation above that of the wild-type LXRE. Surprisingly, however, changing both half-sites to the consensus DR-4 resulted in relatively poor activation (Fig. 2, sequence 5). As we have noted previously (Willy et al. 1995), the weak activation on the consensus DR-4 is not attributable to a notable difference in the DNAbinding affinity of the RXR-LXR heterodimer.

Besides nucleotide substitutions in the half-sites, the spacer and flanking nucleotides were also changed to determine their effect on binding and activation. As can be seen in Figure 2, alterations in the nucleotides flanking the half-sites resulted in lower levels of activation for some response elements (sequences 6-9, 12, and 13), and complete loss of activation for others (sequences 10 and 11). Changing the sequence of the spacer resulted in either an increase or decrease in activation (sequences 14 
and 15), whereas changing the number of nucleotides in the spacer to a DR-3 or DR-5 created an inactive response element (sequences 16 and 17). EMSA experiments have demonstrated a decreased affinity by RXRLXR for some, but not all of these sequences (Willy et al. 1995; data not shown). For example, sequence 11, which is inactive, binds the RXR-LXR heterodimer with high affinity (Willy et al. 1995). These results indicate that the inability to activate is not simply a result of a lower affinity for the element, but involves other critical interactions that are altered when RXR-LXR heterodimers bind to certain sequences.

Although no strict consensus sequence can be defined, these data clearly demonstrate that the LXRE is not the only sequence that can confer retinoid responsiveness to the RXR-LXR heterodimer, and, in fact, several nucleotide substitutions in the $5^{\prime}$ half-site of the LXRE gave enhanced activation by the heterodimer. Our data also suggest that RXR-LXR binding to some DNA sequences does not necessarily equate with activation in response to retinoids.

The LXR AF-2 domain is necessary for RXR-LXR activation by retinoids on an LXRE

The carboxy-terminal region of most nuclear receptors contains a conserved transcriptional activation function (AF-2) that is required for ligand-dependent activation (Fig. 3A) (Danielian et al. 1992). For all characterized RXR heterodimers (i.e., RXR-RAR and RXR-TR), the AF-2 domain of the ligand-binding partner is absolutely required for ligand-dependent activation. Interestingly, in most of these heterodimer complexes, the AF-2 domain of the non-ligand-binding partner (i.e., RXR) is not required and can be mutated or deleted without complete loss of function (Durand et al. 1994; Zhang et al. 1994). To determine whether the AF-2 domain of the ligand-binding partner ( $R X R$ ) contributes to the liganddependent transcriptional activation of the RXR-LXR heterodimer, the RXR AF-2 domain was mutated and tested in transient transfections with LXR for retinoiddependent transactivation. Site-directed mutagenesis was used to generate two RXR AF-2 mutants, RXRL451A, in which leucine 451 was replaced with an alanine, and a double mutant, RXR-L455A/E456A, in which both leucine 455 and glutamic acid 456 were replaced with alanines. Based on previous studies, these RXR mutants should retain both ligand-binding and dimerization abilities (Schulman et al. 1995; data not shown|. However, consistent with previous work (Zhang et al. 1994; Leng et al. 1995; Schulman et al. 1995), RXR AF-2 mutants are not capable of ligand-activated transcription of a reporter containing an RXR response element (TK-CRBPII-LUC) (Fig. 3B). Surprisingly, when the RXR AF-2 mutants were tested with LXR on the LXRE response element, retinoid-dependent induction of the reporter gene was still observed (Fig. 3B). The overall level of activation for wild-type RXR was slightly higher than for the AF-2 mutants, although the fold activation remained similar, suggesting that the RXR AF-2 domain
A

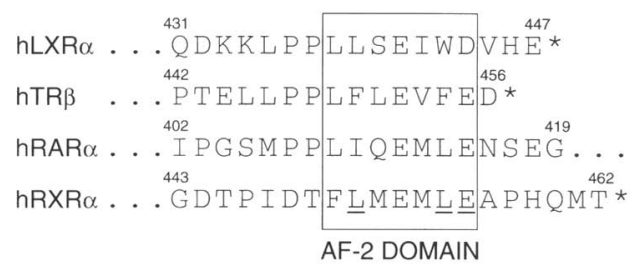

B

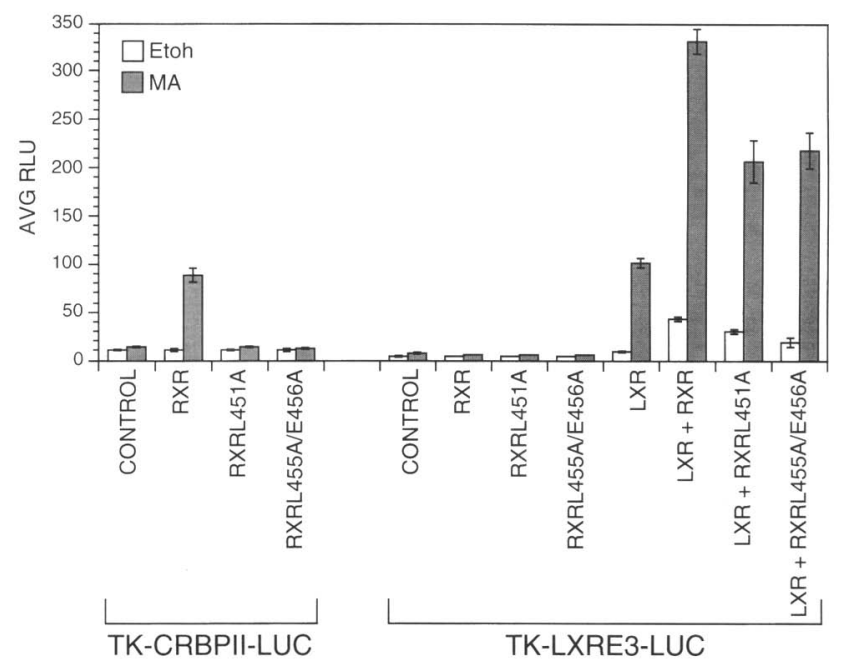

Figure 3. The RXR AF-2 domain is not required for retinoidinduced activation on the LXRE. $\{A\}$ Comparison of the amino acid sequences of AF-2 domains from several RXR dimerization partners (Weinberger et al. 1986; Giguère et al. 1987; Mangelsdorf et al. 1990; Willy et al. 1995). Amino acids in $\mathrm{hRXR} \alpha$ that were mutated to alanines to generate the RXR AF- $2 \mathrm{mu}-$ tants are underlined. (B) Transient transfections in CV-1 cells using wild-type RXR and RXR AF-2 mutants alone or in combination with LXR. The luciferase reporters used (TK-CRBPIILUC for RXR homodimers and TK-LXRE3-LUC for RXR-LXR heterodimers) are shown. In transfections containing both LXR and RXR constructs, one-tenth as much RXR or RXR AF-2 mutant plasmid was used. Bars as in Fig. 1.

has more of an effect on the basal than the ligand-inducible transcriptional response. These results indicate that the RXR AF- 2 domain contributes to, but is not required for, retinoid induction of the RXR-LXR heterodimer.

The above results imply that the AF-2 domain of LXR may contribute to the ligand-dependent transcriptional activation of the heterodimer on an LXRE. To address this possibility, the carboxy-terminal portion of LXR corresponding to the region in and around the putative AF-2 domain was characterized by alanine scanning mutagenesis (Fig. 4A). Five consecutive amino acids of LXR were systematically changed to alanine residues and the resulting LXR mutants were tested for activation by the RXR-selective ligand methoprene acid. Retinoid inducibility did not vary dramatically when any of the last three amino acids that are outside of the putative AF-2 region were mutated to alanines (Fig. 4A). However, mutations to the tryptophan at position 443 and the aspartic 
A

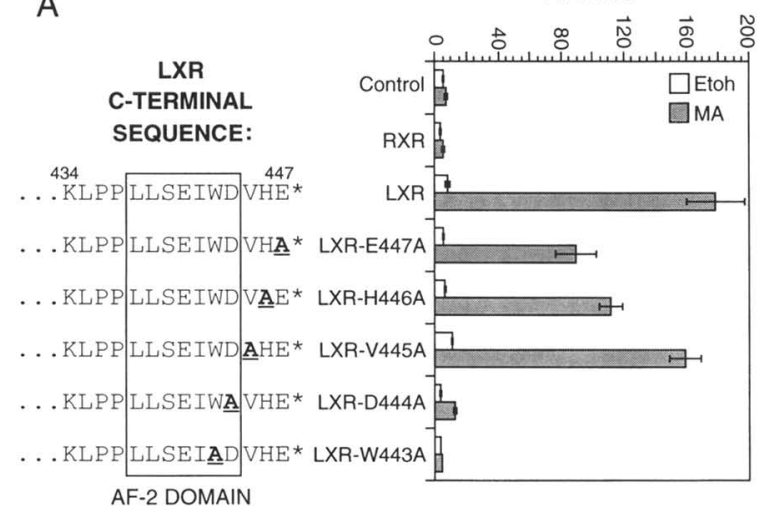

B

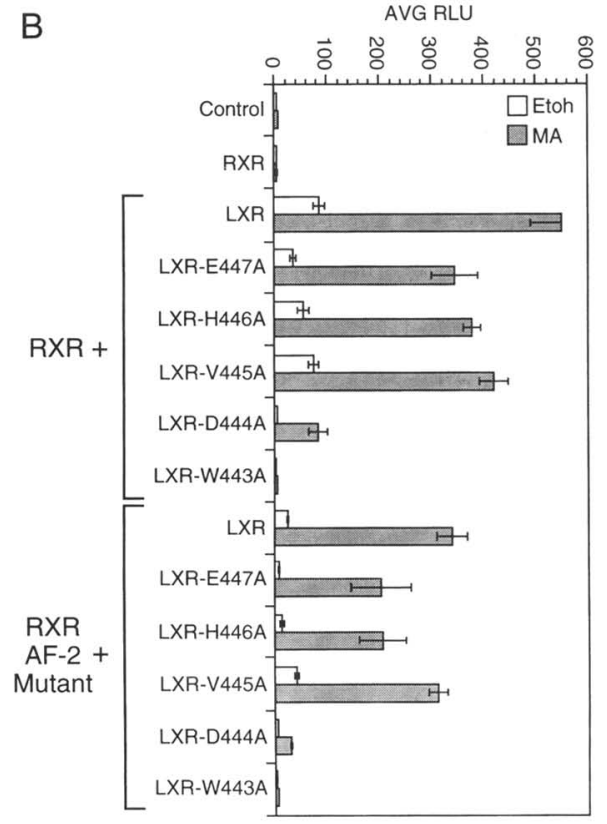

Figure 4. Retinoid-induced activation of the RXR-LXR heterodimer requires the LXR AF-2 domain. $(A$, left $\mid$ Amino acid sequence of the wild-type LXR and LXR AF-2 domain mutants. Amino acids that were mutated to alanines are underlined. (Right) Transient transfections of CV-1 cells with the LXR AF- 2 mutant receptors shown using the TK-LXRE3-LUC reporter. $(B)$ Transient transfections of CV-1 cells using LXR and the LXR AF-2 mutant receptors in combination with RXR or the RXR AF-2 mutant, RXR-L455A/E456A. In transfections containing both LXR and RXR constructs, one-tenth as much RXR or its mutant plasmid was used. $(A, B)$ Bars as in Fig. 1.

acid at 444 indicate that both are required for full transcriptional activation (Fig. 4A). Whereas the LXRW443A mutant exhibited no detectable activation under any conditions tested, the LXR-D444A mutant was able to confer a low level of activation that increased slightly when RXR was also overexpressed (Fig. 4B). Overexpressed RXR could not rescue the LXR-W443A mutant under any conditions, indicating a crucial role for this amino acid in transcriptional activation. Consistent with this finding, when tryptophan 443 was changed to a leucine, the resulting mutant was also inactive under all conditions tested (data not shown). Because the mutated amino acids in LXR that affect activation are contained within the region that corresponds to the conserved activation domain in RXR and other receptors (Danielian et al. 1992), we designate this region as the LXR AF-2 domain (see Fig. 4A). As was the case with wild-type LXR (Fig. 3B), a similar response was seen with the LXR mutants regardless of whether RXR or the RXR AF-2 mutant were also expressed (Fig. 4B). In vitro transcription/translation and EMSA analysis of LXR AF-2 mutants showed that all were made in equivalent amounts to wild-type LXR and all exhibited comparable DNA binding as compared with wild-type LXR /data not shown). Transient transfection experiments with wildtype LXR showed a decrease in reporter activation by methoprene acid when increasing amounts of LXRW443A were added, demonstrating that the LXRW443A protein was expressed in transfected cells. Western blot analysis of LXR and LXR AF-2 mutants ex- pressed in transfected cells also indicated that wild-type and mutant proteins were expressed at comparable levels (data not shown).

Although we have shown previously that methoprene acid is strictly an RXR-selective ligand (Harmon et al. 1995), the possibility existed that the observed effects on the RXR-LXR heterodimer were in some way peculiar to methoprene acid. To rule out this possibility, dose response experiments (Fig. 5A-F) were performed with methoprene acid and other known RXR ligands, including the natural ligand 9-cis retinoic acid and the potent synthetic ligand LG69 (Boehm et al. 1994). Using both RXR and LXR AF- 2 mutants, these experiments recapitulated completely the results seen with methoprene acid alone (Fig. 4), confirming that the AF-2 domain of LXR, but not $\mathrm{RXR}$, is required for activation of the heterodimer. Significantly, the dose responses for these ligands on the wild-type RXR-LXR heterodimer are identical to those observed on the wild-type RXR homodimer (Boehm et al. 1994; Harmon et al. 1995; Willy et al. 1995), implying that in both cases RXR alone binds ligand.

To further demonstrate that the LXR AF-2 domain is required for activation of the RXR-LXR heterodimer, trans-activation assays were performed with DNA-binding orientation-independent heterodimers. For these experiments, GAL4-chimeric receptors were used with a luciferase reporter containing four copies of the GAL4 DNA-binding site (TK-MH100X4-LUC). A chimeric receptor containing the amino terminus and DNA-binding domain of GAL4 fused to the RXR ligand-binding do- 

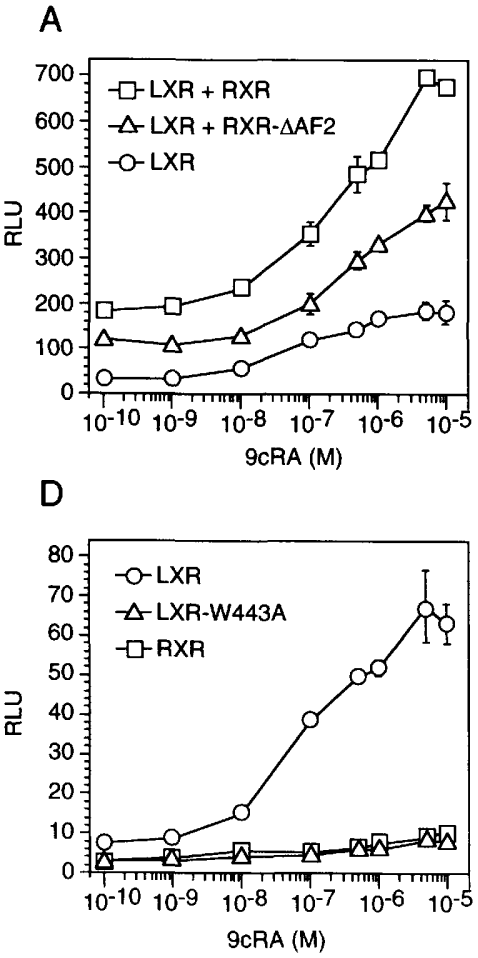

B

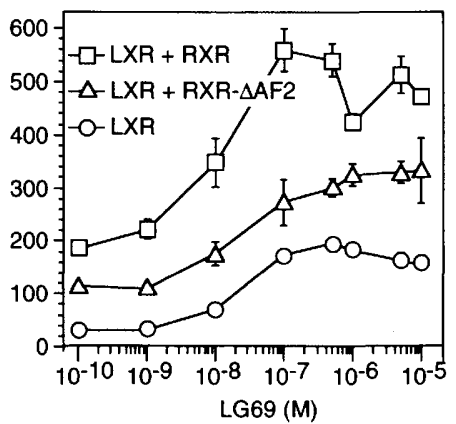

E

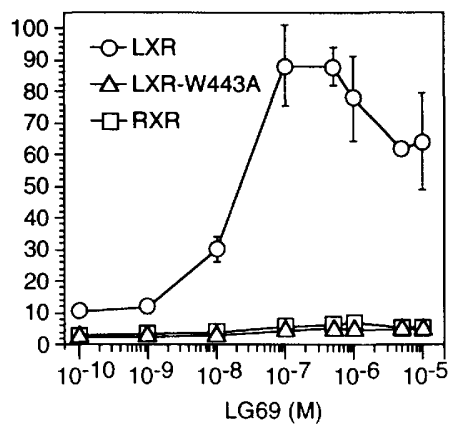

C

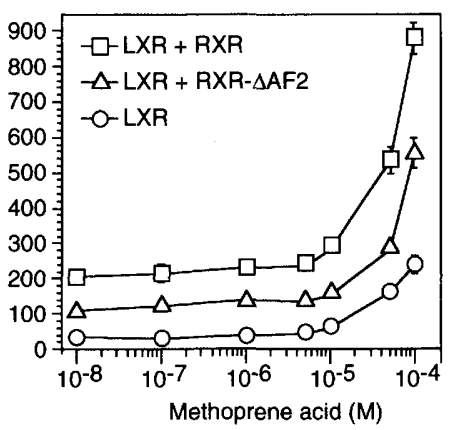

$\mathrm{F}$

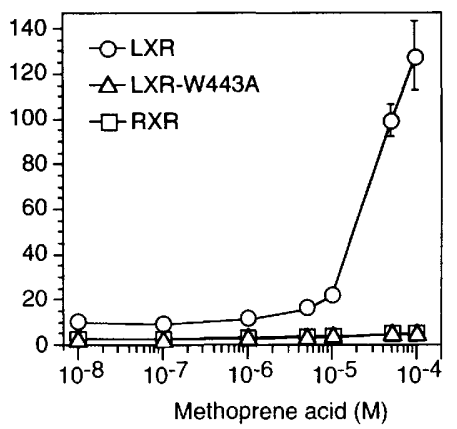

Figure 5. The LXR AF-2 domain is required for RXR-LXR trans-activation by several different RXR ligands. CV-1 cells were transiently transfected with a TK-LXRE3-LUC reporter and the CMX receptor expression plasmids indicated in $A-F$. Cells were then exposed to increasing concentrations of the indicated ligands and analyzed for luciferase activity. In transfections containing both LXR and RXR, one-tenth as much RXR or RXR mutant plasmid was used. RXR- $A$ AF2 represents CMX-RXR-L455A/E456A. (RLU) Relative light units.

main with the L455A/E456A AF-2 mutation /GAL4RXR- $\triangle A F 2$ | was tested for its ability to activate transcription in response to ligand in the presence or absence of exogenous LXR, LXR-W443A, or TR. As seen in Figure 6, GAL4-RXR strongly activated the reporter in response to methoprene acid, whereas activation by the GAL4-RXR AF-2 mutant was greatly reduced. However, ligand-induced activation of the GAL4-RXR AF-2 mutant could be increased significantly when LXR, but not the LXR AF-2 mutant (LXR-W443A) or TR, were added in a dose-dependent manner. These results indicate that the LXR AF-2 domain can replace the nonfunctional RXR AF-2 domain and restore RXR ligand activation. The ability of LXR to enable RXR ligand activation in the context of the orientation-independent GAL4 system is also further evidence that the position of the RXR in the heterodimer is not the only determining factor for RXR ligand activation.

The results from experiments outlined in Figures 3-6 demonstrate that the AF-2 domain of LXR is necessary for retinoid-dependent transcriptional activation by the RXR-LXR heterodimer. Therefore, the RXR-LXR heterodimer is unique in that the AF-2 domain of the nonligand-binding partner (LXR) is required for ligand-dependent trans-activation, whereas the AF- 2 domain of the ligand-binding partner (RXR) is not.

\section{Discussion}

In this paper we have defined several properties of the RXR-LXR heterodimer that distinguish it from other RXR heterodimer signaling pathways. One conserved feature of RXR heterodimers with RAR, TR, and VDR that sets them apart from the RXR-LXR heterodimer is the inability of these other complexes to be activated from the basal state by RXR ligands. In the case of at least one of these complexes, the RXR-RAR heterodimer, Glass and coworkers have shown that the inability of RXR to bind ligand is a result of an allosteric block imposed by RAR (Kurokawa et al. 1994). Thus, activation of these heterodimers is accomplished by ligand binding to the receptor partner of RXR, which exclusively occupies the $3^{\prime}$ half-site of the direct repeat response element (Kurokawa et al. 1993; Perlmann et al. 1993; Schräder et al. 1995). In the one known example (RAR-RXR) where this polarity is switched and the partner of RXR occupies the $5^{\prime}$ half-site, the heterodimer is no longer ligand-activated (Kurokawa et al. 1994). One interpretation of these results is that the ligand inducibility of a receptor is determined in part by its orientation on DNA. In this work, we have shown that the polarity of the RXR-LXR heterodimer, although similar to that of other RXR heterodimers, is unique in that the 


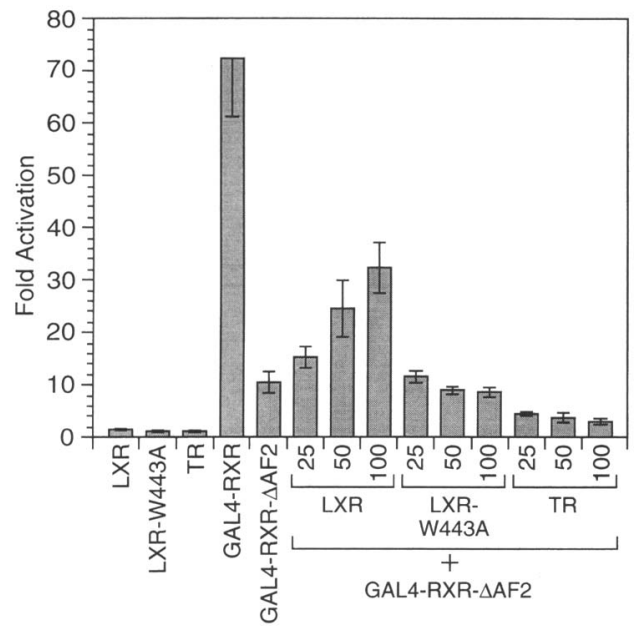

Figure 6. Polarity-independent trans-activation of the RXRLXR heterodimer requires a functional LXR AF-2 domain. CV-1 cells were transiently transfected with a luciferase reporter containing GAL4 DNA-binding sites and the indicated CMX-receptor expression plasmids. Each transfection contained $25 \mathrm{ng}$ of CMX-GAL4-chimeric receptor alone or with 25,50 , or 100 ng of CMX-LXR, CMX-LXR-W443A, or CMX-TR $\beta$ expression plasmid. The LXR, LXR-W443A, and TR controls (first three lanes) contained $100 \mathrm{ng}$ of receptor expression plasmid and 25 ng of CMX-GAL4 ${ }_{\text {DBD }}$. GAL4-RXR- $\triangle A F 2$ represents CMXGAL4-RXR LBD $-\mathrm{L} 455 \mathrm{~A} / \mathrm{E} 456 \mathrm{~A}$. The methoprene acid response is expressed as the fold activation of triplicate assays \pm S.E.

ligand-binding partner (RXR) occupies the $5^{\prime}$ position of the response element, whereas the partner that contributes to DNA-binding specificity (LXR) occupies the $3^{\prime}$ half-site. Similar results have also been found with the orphan receptors NGFI-B and NURR1, which can bind specific response elements as monomers or as heterodimers with RXR, provided that NGFI-B occupies the $3^{\prime}$ half-site of the response element (Perlmann and Jansson 1995). As RXR heterodimers, these orphan receptors can also direct retinoid-dependent transcriptional activation. The ability of the $3^{\prime}$ partner to enable RXR to bind and be activated by ligand appears to be a unique feature of these receptors and further strengthens the notion that one function of orphan receptors such as LXR and NGFI-B is to increase the complexity of 9cRA signaling through RXR (Leblanc and Stunnenberg 1995). From these observations, we conclude that RXR ligand-activation potential is dictated not solely by its position on DNA, but rather by other factors such as the receptor partner and sequence of the response element. In further agreement with this conclusion, we note that RXR-LXR heterodimers bind with the same fixed polarity to LXREs (which are retinoid-responsive) and canonical DR4 elements (which are not retinoid-responsive). In addition, RXR-TR heterodimers, which are not responsive to RXR ligands, bind with the same fixed polarity to all DR4 elements, including LXRE sequences (Perlmann et al. 1993; P. Willy, unpubl.). Finally, polarity-independent experiments using GAL4-RXR-LXR heterodimers and their AF-2 mutants (Fig. 6) further emphasize the importance of the partner receptor in mediating the RXR ligand response.

This work has also identified critical nucleotides in the LXRE sequence that contribute to activation by the heterodimer. From this analysis it is clear that the LXRE sequence is not the only element capable of mediating the RXR-LXR response. Indeed, mutagenesis has defined a sequence that as a single copy gives a twofold greater activation than the original LXRE sequence (Fig. 2, sequence 2). Nevertheless, it has not been possible to generate a consensus LXRE sequence, because several individual changes that increase activity do not act cooperatively (or even additively) when combined. For example, changing either half-site to the canonical AGGTCA sequence results in an increase in retinoid activation. However, altering both half-sites to AGGTCA lowers activity dramatically. Consistent with other receptor response elements (Umesono et al. 1991; Mader et al. 1993; Katz and Koenig 1994), in addition to the half-site sequences, both the spacing and flanking nucleotides of the LXRE are important for binding and activation. The LXRE has an absolute spacing requirement of four nucleotides (Fig. 2), the sequence of which can modulate, but is not critical, for activation. The nucleotides immediately upstream and downstream of the core element are also important for activation but are less critical for in vitro DNA binding.

In addition to the contributions made by the LXR ligand- and DNA-binding domains as well as the response element, a unique mechanism of ligand-dependent transcriptional activation through the RXR-LXR heterodimer has been identified. Our results demonstrate that the functional AF-2 domain of LXR is critical for retinoid activation of the RXR-LXR heterodimer. Surprisingly, the AF-2 domain of RXR, the ligand-binding receptor in this heterodimer, is not required for activation and its apparent contribution is minimal. These results differ from previous observations with RXR-TR and RXRRAR heterodimers in several ways. In these heterodimers the AF-2 domain of the ligand-binding receptor is required for ligand-dependent transcriptional activation (Saatcioglu et al. 1993; Barettino et al. 1994; Durand et al. 1994; Tone et al. 1994; Baniahmad et al. 1995). With these other receptors it has therefore been suggested that ligand binding to the receptor induces a conformational change (Renaud et al. 1995) that dissociates a corepressor (Chen and Evans 1995; Hörlein et al. 1995) and attracts a coactivator (Oñate et al. 1995), thereby permitting positive interactions with components of the transcriptional machinery (Fig. 7A). Together with the ability of at least one of these receptors (i.e., RAR) to allosterically block RXR ligand binding, these observations likely explain why RXR cannot respond to retinoids in the context of these heterodimeric interactions (Kurokawa et al. 1995). Our results suggest a different mechanism of action for the RXR-LXR heterodimer (Fig. 7B), namely that the entire heterodimer complex, not simply the ligand-binding partner, must undergo a conformational change that allows transcrip- 
A
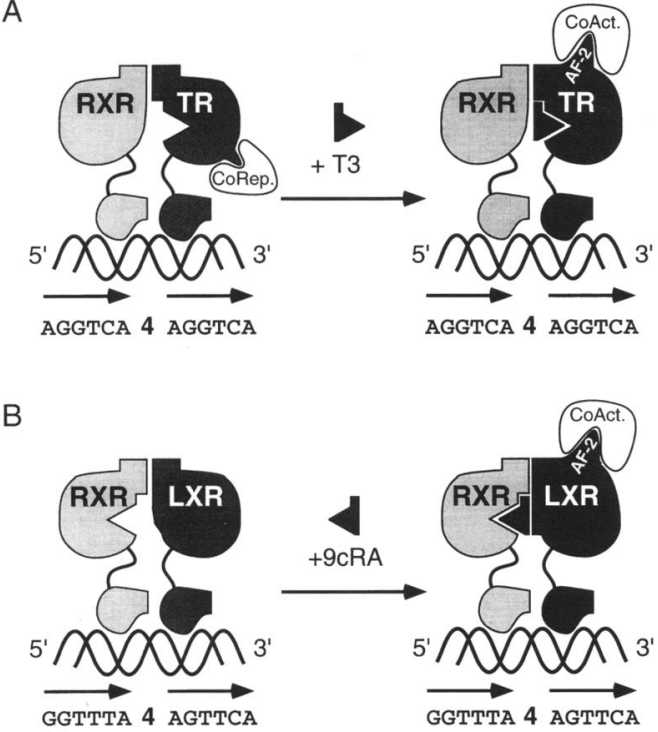

Figure 7. Models for two mechanisms of ligand activation of RXR heterodimers. (A) In many RXR heterodimers, RXR cannot be activated by $9 \mathrm{cRA}$. In the basal state, the $3^{\prime}$ receptor partner (e.g., TR) binds a corepressor (CoRep.) that suppresses promoter activity. Ligand binding to the $3^{\prime}$ partner induces a conformational change in the receptor that simultaneously releases the corepressor and alters the activation domain (AF-2) for interaction with coactivator (CoAct.) proteins. $(B)$ In the RXR-LXR heterodimer, basal transcription is not repressed and RXR ligand binding is enabled while RXR maintains its position on the 5 ' half-site. The ligand-mediated conformational change in RXR induces a change in LXR, which can then interact with putative coactivators to affect transcription. In both $A$ and $B$ ligand-induced trans-activation requires the AF- 2 of the $3^{\prime}$ receptor partner, regardless of which partner binds ligand.

tional activation to occur. In this model, one receptor binds ligand (RXR), whereas the AF- 2 domain of the other receptor (LXR) is exposed, thereby allowing an interaction with factors that affect transcription. Interestingly, unlike RXR-TR and RXR-RAR, in the absence of ligand there is no observable repression with RXR-LXR, suggesting that corepressor may not bind the RXR-LXR heterodimer. Consistent with this idea, the overexpression of RXR and LXR together results in a significant increase in the basal activity (Willy et al. 1995; this work). Because mutation of the LXR AF- 2 domain results in complete lack of activation, one of the likely requirements for ligand induction is association of a coactivator with LXR (Fig. 7B). However, the possibility that the LXR AF-2 mutant becomes inactive because it gains the novel ability to interact with a dominant repressor cannot be ruled out at the present time. Future experiments will address the nature of the interaction of LXR with coregulator molecules.

In conclusion, retinoid signaling through the RXRLXR heterodimer differs mechanistically from other heterodimer signaling pathways. In the RXR-LXR heterodimer, RXR binds ligand while occupying the $5^{\prime}$ half-site of a specific subset of DR4-type response elements.
Nucleotides in the response element dictate whether or not binding is coupled to activation by the heterodimer. Ligand binding by RXR then confers a change in LXR, allowing for transcriptional activation that is mediated through the LXR AF-2 domain. This finding suggests the intriguing possibility that this novel mechanism of activation through the AF-2 domain of the non-ligand-binding partner may be used by other receptor heterodimers that work through RXR signaling.

\section{Materials and methods}

\section{Plasmids}

Constructions of wild-type receptors inserted into the expression vector PCMX have been detailed elsewhere (Umesono et al. 1991; Willy et al. 1995). CMX-LXRpg and RXR AF-2 mutants were generated by site-directed mutagenesis using the Morph Mutagenesis Kit (5 Prime $\rightarrow 3$ Prime, Inc., Boulder, CO). CMXLXR-AF-2 alanine scanning mutants were generated as follows: mutant oligonucleotides encoding the last five amino acids of LXR with single alanine substitutions were ligated into CMXhLXR $\alpha$ that had been digested with $B g I I I$ and NheI to remove nucleotides encoding the last five amino acids and 3 '-untranslated sequence. Luciferase reporter plasmids were constructed by ligating the appropriate double-stranded oligonucleotides into the HindIII site of the TK-LUC vector. TK-LXRE3-LUC (Willy et al. 1995), TK-CRBPII-LUC (Mangelsdorf et al. 1991), TK-MH100X4-LUC, and CMX-GAL4-hRXR $\alpha$ (Harmon et al. 1995| have been described. The sequences for L4G and G4L reporters are shown in Figure 1 and contain HindIII ends for cloning. TK-L4G-LUC contains three copies of the response element, which are all in a $5^{\prime} \rightarrow 3^{\prime}$ orientation with respect to the transcription start site. TK-G4L-LUC also contains three copies of the response element, with the first two copies in the $5^{\prime} \rightarrow 3^{\prime}$ direction and the last in the opposite orientation. LXRE mutants were cloned into TK-LUC as a single copy in the $5^{\prime} \rightarrow 3^{\prime}$ orientation with respect to the transcription start site. All plasmid constructs and mutations were verified by sequencing.

\section{EMSAS}

Receptor proteins were generated using coupled in vitro transcription/translation as per manufacturer's instructions (Promega). Sequences of double-stranded oligonucleotides were as shown in the figures and text and were synthesized with HindIII overhangs. Binding reactions were performed in a total volume of $20 \mu \mathrm{l}$ consisting of $75 \mathrm{~mm} \mathrm{KCl}, 20 \mathrm{~mm}$ HEPES at pH 7.4, $2 \mathrm{~mm}$ DTT, $7.5 \%$ glycerol, $0.1 \%$ NP-40, $2 \mu$ g of poly[d(I-C)] (Pharmacia), 60 pmoles of a nonspecific single-stranded oligonucleotide (for removal of nonspecific binding in the lysates), and $2 \mu \mathrm{l}$ of each receptor lysate or unprogrammed (control) lysates to give a total of $4 \mu \mathrm{l}$ of lysate per reaction. Binding reactions were incubated for $\sim 90 \mathrm{~min}$ on ice followed by the addition of 40 fmoles of ${ }^{32} \mathrm{P}$-labeled oligonucleotide probe (labeled by end-filling) and incubated further for $30 \mathrm{~min}$ at room temperature. Samples were analyzed on $5 \%$ polyacrylamide gels run in $0.5 \times \mathrm{TBE}$ buffer at $275 \mathrm{~V}$ for $1.5 \mathrm{hr}$.

\section{Cell culture and cotransfection assays}

$\mathrm{CV}-1$ cells were maintained at $37^{\circ} \mathrm{C}, 7 \% \mathrm{CO}_{2}$ in Dulbecco's modified Eagle medium (DMEM) containing $5 \%$ calf bovine serum (CBS). Transfections were performed in 48-well plates in 
media containing $5 \%$ dextran-charcoal stripped CBS by the calcium phosphate coprecipitation technique as described previously (Willy et al. 1995). Eight hours after transfections ligands were delivered to cells at $10^{3}$-fold dilutions $[0.1 \%$ (vol $/ \mathrm{vol})$ of solvent in medial. Retinoids were manipulated under gold light and stored dark in ethanol or methanol under nitrogen gas at $-80^{\circ} \mathrm{C}$. Cells were harvested $36 \mathrm{hr}$ after addition of ligand and analyzed for luciferase and $\beta$-galactosidase activity using a Dynatech ML3000 luminometer and MR5000 spectrophotometer, respectively. For most experiments, cotransfection of DNA into mammalian cells was accomplished with $50 \mathrm{ng}$ reporter plas-

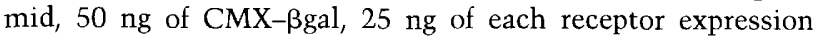
plasmid, and pGEM carrier to give $375 \mathrm{ng}$ of DNA/well /see figure legends for details on individual experiments|. Transfections with mutant LXRE reporters (Fig. 2) contained $65 \mathrm{ng}$ of receptor, $25 \mathrm{ng}$ of reporter, $50 \mathrm{ng}$ of CMX- $\beta$ gal, and pGEM carrier to give $375 \mathrm{ng}$ of $\mathrm{DNA} /$ well. For controls, CMX vector was used in place of the receptor plasmid. Ligand concentrations were $10^{-4} \mathrm{M}$ methoprene acid and $10^{-6} \mathrm{M}$ thyroid hormone. All transfection data points were normalized using an internal $\beta$-galactosidase marker (Mangelsdorf et al. 1990) and represent the mean of triplicate assays \pm S.E.

\section{Acknowledgments}

We thank Wenzhen Ma and Heather Lawrence for excellent technical assistance and Dr. Daniel Peet and other members of the Mango laboratory for critical discussions. We also thank Drs. Richard Heyman and Marcus Boehm (Ligand Pharmaceuticals, Inc., San Diego, CA) for methoprene acid and LG69; and Dr. Ronald Evans for the hTR $\beta$ expression plasmid. D.J.M. is an investigator of the Howard Hughes Medical Institute (HHMI) at the University of Texas Southwestern Medical Center. This work was supported by HHMI, a grant from the Welch Foundation, and a National Institutes of Health Pharmacological Sciences Training Grant.

The publication costs of this article were defrayed in part by payment of page charges. This article must therefore be hereby marked "advertisement" in accordance with 18 USC section 1734 solely to indicate this fact.

\section{References}

Baniahmad, A., X. Leng, T.P. Burris, S.Y. Tsai, M.-J. Tsai, and B.W. O'Malley. 1995. The $\tau 4$ activation domain of the thyroid hormone receptor is required for release of a putative corepressor(s) necessary for transcriptional silencing. Mol. Cell. Biol, 15: 76-86.

Barettino, D., M.D.M.V. Ruiz, and H.G. Stunnenberg. 1994. Characterization of the ligand-dependent transactivation domain of thyroid hormone receptor. EMBO J. 13: 3039-3049.

Boehm, M.F., L. Zhang, B.B. Badea, S.K. White, D.E. Mais, E. Berger, C.M. Suto, M.E. Goldman, and R.A. Heyman. 1994. Synthesis and structure-activity relationships of novel retinoid X receptor-selective retinoids. J. Med. Chem. 37: 29302941.

Cavaillès, V., S. Cauvois, F. L'Horset, G. Lopez, S. Hoare, P.J. Kushner, and M.G. Parker. 1995. Nuclear factor RIP140 modulates transcriptional activation by the estrogen receptor. EMBO T. 14: 3741-3751.

Chen, J.D. and R.M. Evans. 1995. A transcriptional co-repressor that interacts with nuclear hormone receptors. Nature 377: 454-457.

Danielian, P.S., R. White, J.A. Lees, and M.G. Parker. 1992. Identification of a conserved region required for hormone dependent transcriptional activation by steroid hormone receptors. EMBO I. 11: 1025-1033.

Durand, B., M. Saunders, C. Gaudon, B. Roy, R. Losson, and P. Chambon. 1994. Activation function 2(AF-2) of retinoic acid receptor and 9-cis retinoic acid receptor: Presence of a conserved autonomous constitutive activating domain and influence of the nature of the response element on AF-2 activity. $E M B O ~ J .13: 5370-5382$.

Forman, B.M., K. Umesono, J. Chen, and R.M. Evans. 1995. Unique response pathways are established by allosteric interactions among nuclear hormone receptors. Cell 81: 541550.

Giguère, V., E.S. Ong, P. Segui, and R.M. Evans. 1987. Identification of a receptor for the morphogen retinoic acid. Nature 330: 624-629.

Halachmi, S., E. Marden, G. Martin, H. MacKay, C. Abbondanza, and M. Brown. 1994. Estrogen receptor-associated proteins: Possible mediators of hormone-induced transcription. Science 264: 1455-1458.

Harmon, M.A., M.F. Boehm, R.A. Heyman, and D.J. Mangelsdorf. 1995. Activation of mammalian retinoid X receptors by the insect growth regulator methoprene. Proc. Natl. Acad. Sci. 92: 6157-6160.

Hollenberg, S.M. and R.M. Evans. 1988. Multiple and cooperative trans-activation domains of the human glucocorticoid receptor. Cell 55: 899-906.

Hörlein, A.J., A.M. Näär, T. Heinzel, J. Torchia, B. Gloss, R. Kurokawa, A. Ryan, Y. Kamei, M. Söderström, C.K. Glass, and M.G. Rosenfeld. 1995. Ligand-independent repression by the thyroid hormone receptor mediated by a nuclear receptor co-repressor. Nature 377: 397-404.

Katz, R.W. and R.J. Koenig. 1994. Nucleotide substitutions differentially affect direct repeat and palindromic thyroid hormone response elements. J. Biol. Chem. 269: 9500-9505.

Kurokawa, R., V.C. Yu, A. Näär, S. Kyakumoto, Z. Han, S. Silverman, M.G. Rosenfeld, and C.K. Glass. 1993. Differential orientations of the DNA-binding domain and carboxy-terminal dimerization interface regulate binding site selection by nuclear receptor heterodimers. Genes \& Dev. 7: 1423-1435.

Kurokawa, R., J. DiRenzo, M. Boehm, J. Sugarman, B. Gloss, M.G. Rosenfeld, R.A. Heyman, and C.K. Glass. 1994. Regulation of retinoid signalling by receptor polarity and allosteric control of ligand binding. Nature 371: 528-531.

Kurokawa, R., M. Söderström, A.J. Hörlein, S. Halachmi, M. Brown, M.G. Rosenfeld, and C.K. Glass. 1995. Polarity-specific activities of retinoic acid receptors determined by a co-repressor. Nature 377: 451-454.

Le Douarin, B., C. Zechel, I.M. Garnier, Y. Lutz, L. Tora, B. Pierrat, D. Heery, H. Gronemeyer, P. Chambon, and R. Losson. 1995. The $\mathrm{N}$-terminal part of TIF1, a putative mediator of the ligand-dependent activation function (AF-2) of nuclear receptors, is fused to B-raf in the oncogenic protein $\mathrm{T} 18$. EMBO J. 14: 2020-2033.

Leblanc, B.P. and H.G. Stunnenberg. 1995. 9-Cis retinoic acid signaling: Changing partners causes some excitement. Genes \& Dev. 9: 1811-1816.

Lee, J.W., F. Ryan, J.C. Swaffield, S.A. Johnston, and D.D. Moore. 1995. Interaction of thyroid-hormone receptor with a conserved transcriptional mediator. Nature 374: 91-94.

Leng, X., J. Blanco, S.Y. Tsai, K. Ozato, B.W. O'Malley, and M.-J. Tsai. 1995. Mouse retinoid X receptor contains a separable ligand-binding and transactivation domain in its $\mathrm{E}$ region. Mol. Cell. Biol. 15: 255-263.

Mader, S., P. Leroy, J.-Y. Chen, and P. Chambon. 1993. Multiple parameters control the selectivity of nuclear receptors for their response elements: Selectivity and promiscuity in re- 
sponse element recognition by retinoic acid receptors and retinoid X receptors. J. Biol. Chem. 268: 591-600.

Mangelsdorf, D.J. and R.M. Evans. 1995. The RXR heterodimers and orphan receptors. Cell 83: 841-850.

Mangelsdorf, D.J., E.S. Ong, J.A. Dyck, and R.M. Evans. 1990. Nuclear receptor that identifies a novel retinoic acid response pathway. Nature 345: 224-229.

Mangelsdorf, D.J., K. Umesono, S.A. Kliewer, U. Borgmeyer, E.S. Ong, and R.M. Evans. 1991. A direct repeat in the cellular retinol-binding protein type II gene confers differential regulation by RXR and RAR. Cell 66: 555-561.

Mangelsdorf, D.)., C. Thummel, M. Beato, P. Herrlich, G. Schütz, K. Umesono, B. Blumberg, P. Kastner, M. Mark, P. Chambon, and R.M. Evans. 1995. The nuclear receptor superfamily: The second decade. Cell 83: 835-839.

McKnight, S.L. 1996. Transcription revisited: A commentary on the 1995 Cold Spring Harbor laboratory meeting, "Mechanisms of eukaryotic transcription." Genes \& Dev. 10: 367381.

Nagpal, S., M. Saunders, P. Kastner, B. Durand, H. Nakshatri, and P. Chambon. 1992. Promoter context- and response element-dependent specificity of the transcriptional activation and modulating functions of retinoic acid receptors. Cell 70: 1007-1019.

Nagpal, S., S. Friant, H. Nakshatri, and P. Chambon. 1993. RARs and RXRs: Evidence for two autonomous transactivation functions (AF-1 and AF-2) and heterodimerization in vivo. EMBO T. 12: 2349-2360.

Oñate, S.A., S.Y. Tsai, M.J. Tsai, and B.W. O'Malley. 1995. Sequence and characterization of a coactivator for the steroid hormone receptor superfamily. Science 270: 1354-1357.

Perlmann, T. and L. Jansson. 1995. A novel pathway for vitamin A signaling mediated by RXR heterodimerization with NGFI-B and NURR1. Genes \& Dev. 9: 769-782.

Perlmann, T., P.N. Rangarajan, K. Umesono, and R.M. Evans. 1993. Determinants for selective RAR and TR recognition of direct repeat HREs. Genes \& Dev. 7: 1411-1422.

Renaud, J.P., N. Rochel, M. Ruff, V. Vivat, P. Chambon, H. Gronemeyer, and D. Moras. 1995. Crystal structure of the RAR-gamma ligand-binding domain bound to all-trans retinoic acid. Nature 378: 681-689.

Roy, B., R. Taneja, and P. Chambon. 1995. Synergistic activation of retinoic acid (RA)-responsive genes and induction of embryonal carcinoma cell differentiation by an $\mathrm{RA}$ receptor $\alpha($ RAR $\alpha)$-, RAR $\beta$-, or RAR $\gamma$-selective ligand in combination with a retinoid X receptor-specific ligand. Mol. Cell. Biol. 15: 6481-6487.

Saatcioglu, F., P. Bartunek, T. Deng, M. Zenke, and M. Karin. 1993. A conserved C-terminal sequence that is deleted in $\mathrm{v}$-ErbA is essential for the biological activities of c-ErbA/the thyroid hormone receptor). Mol. Cell. Biol. 13: 3675-3685.

Schräder, M., S. Nayeri, J.-P. Kahlen, K.M. Müller, and C. Carlberg. 1995. Natural vitamin $\mathrm{D}_{3}$ response elements formed by inverted palindromes: Polarity-directed ligand sensitivity of vitamin $\mathrm{D}_{3}$ receptor-retinoid X receptor heterodimer-mediated transactivation. Mol. Cell. Biol. 15: 1154-1161.

Schulman, I.G., D. Chakravarti, H. Juguilon, A. Romo, and R.M. Evans. 1995. Interactions between the retinoid X receptor and a conserved region of the TATA-binding protein mediate hormone-dependent transactivation. Proc. Natl. Acad. Sci. 92: 8288-8292.

Strähle, U., G. Kolck, and G. Schütz. 1987. A DNA sequence of 15 base pairs is sufficient to mediate both glucocorticoid and progesterone induction of gene expression. Proc. Natl. Acad. Sci. 84: 7871-7875.
Tone, Y., M.A. Collingwood, M. Adams, and V.K. Chatterjee. 1994. Functional analysis of a transactivation domain in the thyroid hormone $\beta$ receptor. J. Biol. Chem. 269: 3115731161 .

Umesono, K., K.K. Murakami, C.C. Thompson, and R.M. Evans. 1991. Direct repeats as selective response elements for the thyroid hormone, retinoic acid, and vitamin $D_{3}$ receptors. Cell 65: 1255-1266.

vom Baur, E., C. Zechel, D. Heery, M. Heine, J.M. Garnier, V. Vivat, B. Le Douarin, H. Gronemeyer, P. Chambon, and R. Losson. 1996. Differential ligand-dependent interactions between the AF-2 activating domain of nuclear receptors and the putative transcriptional intermediary factors mSUG1 and TIF1. EMBO J. 15: 110-124.

Webster, N.J.G., S. Green, J.R. Jin, and P. Chambon. 1988. The hormone-binding domains of the estrogen and glucocorticoid receptors contain an inducible transcription activation function. Cell 54: 199-207.

Weinberger, C., C.C. Thompson, E.S. Ong, R. Lebo, D.J. Gruol, and R.M. Evans. 1986. The c-erb-A gene encodes a thyroid hormone receptor. Nature 324: 641-646.

Willy, P.J., K. Umesono, E.S. Ong, R.M. Evans, R.A. Heyman, and D.J. Mangelsdorf. 1995. LXR, a nuclear receptor that defines a distinct retinoid response pathway. Genes \& Dev. 9: 1033-1045.

Zhang, X.-K., G. Salbert, M.-O. Lee, and M. Pfahl. 1994. Mutations that alter ligand-induced switches and dimerization activities in the retinoid $\mathrm{X}$ receptor. Mol. Cell. Biol. 14: $4311-4323$. 


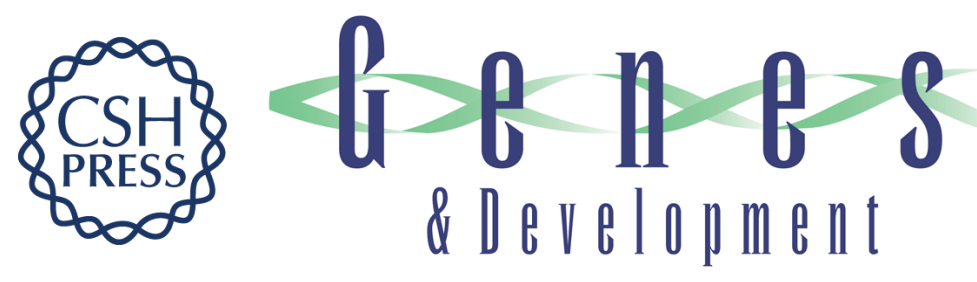

\section{Unique requirements for retinoid-dependent transcriptional activation by the orphan receptor LXR.}

P J Willy and D J Mangelsdorf

Genes Dev. 1997, 11:

Access the most recent version at doi:10.1101/gad.11.3.289

References This article cites 45 articles, 20 of which can be accessed free at: http://genesdev.cshlp.org/content/11/3/289.full.html\#ref-list-1

License

Email Alerting

Service

Receive free email alerts when new articles cite this article - sign up in the box at the top right corner of the article or click here.

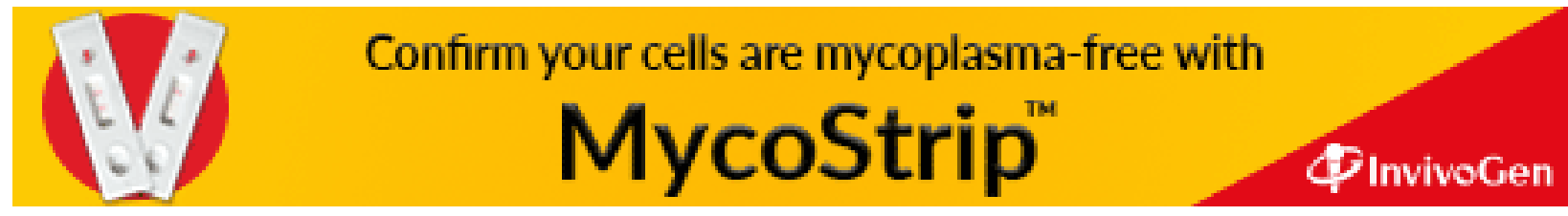

